\title{
Sequence Determinants Spanning -10 Motif and Spacer Region Implicated in Unique Ehrlichia chaffeensis Sigma 32-Dependent Promoter Activity of dnaK Gene
}

\author{
Huitao Liu and Roman R. Ganta* \\ Center of Excellence for Vector-Borne Diseases, Department of Diagnostic Medicine/Pathobiology, College of Veterinary \\ Medicine, Kansas State University, Manhattan, KS, United States
}

OPEN ACCESS

Edited by:

Hari S. Misra,

Bhabha Atomic Research Centre

(BARC), India

Reviewed by:

Jere W. McBride,

The University of Texas Medical

Branch at Galveston, United States

Bing Zhu,

The University of Texas Medical

Branch at Galveston, United States

Tian Luo,

The University of Texas Medical Branch at Galveston, United States

*Correspondence: Roman R. Ganta rganta@vet.k-state.edu

Specialty section:

This article was submitted to Microbial Physiology and Metabolism,

a section of the journa

Frontiers in Microbiology

Received: 10 May 2019

Accepted: 18 July 2019

Published: 02 August 2019

Citation:

Liu H and Ganta RR (2019) Sequence Determinants Spanning

-10 Motif and Spacer Region

Implicated in Unique Ehrlichia chaffeensis Sigma 32-Dependent

Promoter Activity of dnaK Gene.

Front. Microbiol. 10:1772.

doi: 10.3389/fmicb.2019.01772
Ehrlichia chaffeensis is an obligate intracellular tick-borne bacterium that causes human monocytic ehrlichiosis. Studying Ehrlichia gene regulation is challenge, as this and related rickettsiales lack natural plasmids and mutagenesis experiments are of a limited scope. E. chaffeensis contains only two sigma factors, $\sigma^{32}$ and $\sigma^{70}$. We previously developed Escherichia coli surrogate system to study transcriptional regulation from RNA polymerase (RNAP) containing Ehrlichia $\sigma^{32}$ or $\sigma^{70}$. We reported that RNAP binding motifs of $E$. chaffeensis genes recognized by $\sigma^{32}$ or $\sigma^{70}$ share extensive homology and that transcription may be initiated by either one of the sigma factors, although transcriptional efficiencies differ. In the current study, we investigated mapping the E. chaffeensis dnaK gene promoter using the pathogen $\sigma^{32}$ expressed in E. coli lacking its native $\sigma^{32}$. The $E$. coli surrogate system and our previously described in vitro transcription system aided in defining the unique -10 motif and spacer sequence of the dnaK promoter. We also mapped $\sigma^{32}$ amino acids/domains engaged in its promoter regulation in $E$. chaffeensis. The data reported in this study demonstrate that the -10 and -35 motifs and spacer sequence located between the two motifs of dnaK promoter are critical for the RNAP function. Further, we mapped the importance of all six nucleotide positions of the -10 motif and identified critical determinants within it. In addition, we reported that the lack of C-rich sequence upstream to the -10 motif is unique in driving the pathogen-specific transcription by its $\sigma^{32}$ from dnaK gene promoter. This is the first study in defining an E. chaffeensis $\sigma^{32}$-dependent promoter and it offers insights about how this and other related rickettsial pathogens regulate stress response genes.

Keywords: gene regulation, intracellular bacteria, Anaplasmataceae, sigma factor, Ehrlichia chaffeensis, RNA polymerase

\section{INTRODUCTION}

Ehrlichia chaffeensis, a Gram-negative, obligate intracellular tick-borne rickettsial pathogen, causes human monocytic ehrlichiosis (HME) (Dunning Hotopp et al., 2006). HME as an emerging infectious disease, first reported in the United States in 1987, has become one of the most prevalent tick borne diseases in the United States and is also described from several other parts of the world 
(Ismail et al., 2010; Yabsley, 2010). Further, E. chaffeensis infects several other vertebrates, including dogs, goats, coyotes and white-tailed deer (Dawson et al., 1996; Lockhart et al., 1997; Breitschwerdt et al., 1998; Dugan et al., 2000; Kocan et al., 2000; Davidson et al., 2001). The pathogen infection in people may result in an acute flu-like illness with symptoms ranging from persistent fever, headache, myalgia, anorexia and chills (Walker et al., 2008). HME patients may exhibit leukopenia, thrombocytopenia, anemia, and upgraded levels of serum hepatic aminotransferases (Walker et al., 2008). In addition, other related Anaplasmataceae family pathogens included in the genera Ehrlichia and Anaplasma have been established as causative agents of emerging diseases in people and various vertebrate animals in recent years (Walker and Dumler, 1996; Walker et al., 2008; Rikihisa, 2010). Though some progress is made in establishing genetics in E. chaffeensis (Cheng et al., 2013; Wang et al., 2017). and similarly in other related Ehrlichia and Anaplasma (Long et al., 2005; Felsheim et al., 2006; Crosby et al., 2014; Wood et al., 2014; mcClure et al., 2017), the genetic tool kit and its application is still limited. For example, it is not possible to investigate regulation of gene expression by transforming this group of important pathogens, possibly also because the pathogens lack naturally existing extrachromosomal plasmids. This major impediment limits the understanding of molecular mechanisms used by the pathogens in regulating gene expression in support of their continued survival in vertebrate and tick hosts and in causing pathogenesis (Dumler et al., 1993; Davidson et al., 2001; Unver et al., 2002). Several prior studies reported differences in gene expression of E. chaffeensis impacted by different host environments (Seo et al., 2008; Kuriakose et al., 2011). However, it is unclear how the organism regulates its gene expression in support of its adaptation to the hosts.

Regulation of gene expression in bacteria is primarily controlled at the transcription. An RNA polymerase (RNAP) core enzyme with a sigma $(\sigma)$ factor offers a simple and valid mechanism for bacteria to rapidly accommodate to diverse environmental changes by suitably modifying the transcriptional profiles (Gruber and Gross, 2003; Gunesekere et al., 2006; Browning and Busby, 2016). Typically, an RNAP holoenzyme is a multi-subunit complex consists of a core enzyme containing two alpha $(\alpha)$, a beta $(\beta)$, a beta' $\left(\beta^{\prime}\right)$, and a omega $(\omega)$ subunits and then the inclusion of a $\sigma$ factor (Chamberlin et al., 1983). A $\sigma$ factor enables a core enzyme in specific binding to the promoter region of a gene for initiating transcription. Numbers of $\sigma$ factors differ depending on the genome size variations and the environmental diversification of a bacterium (Kill et al., 2005). For example, Escherichia coli possesses seven $\sigma$ factors, while $109 \sigma$ factors are identified in Sorangium cellulosum (Han et al., 2013; Tripathi et al., 2014). Obligate intracellular bacteria generally tend to have reduced genomes and consequently also have fewer $\sigma$ factors (Darby et al., 2007). For example, E. chaffeensis genome of $1,176 \mathrm{~kb}$ has only two $\sigma$ factor genes; $r p o D$ (ECH_0760) (the primary housekeeping $\sigma^{70}$ gene) and $r p o H$ (ECH_0655) (the alternate $\sigma^{32}$ gene) (Dunning Hotopp et al., 2006) (GenBank \# NC_007799.1).
To study gene regulation in E. chaffeensis, we previously described in vitro transcription system and an E. coli surrogate system that is valuable in investigating gene regulation driven from its $\sigma^{70}$ (Faburay et al., 2011; Liu et al., 2013, 2016). Earlier, we also defined the promoters of several pathogen genes by utilizing in vitro transcription assays where E. coli RNAP core enzyme is reconstituted with the recombinant E. chaffeensis $\sigma$ factors (Faburay et al., 2011; Liu et al., 2013). Our studies demonstrated that the RNAP binding motifs ( -10 and -35 regions) of E. chaffeensis gene promoters share extensive homology and that they are recognizable by RNAP with either one of its only two sigma factors; $\sigma^{32}$ or $\sigma^{70}$, although affinities vary for different gene promoters (Liu et al., 2013). In E. coli, gene expression of heat shock proteins; Dnak-DnaJ-GrpE and GroES-GroEL chaperone complexes, are controlled by its $\sigma^{32}$ (Nonaka et al., 2006). Similarly, we discovered that the E. chaffeensis chaperon protein gene (Ech_0471) encoding for DnaK protein is transcribed primarily by $\sigma^{32}$ (Liu et al., 2013). Genes regulated by $\sigma^{32}$ are known to induce cellular responses under varieties of stresses confronted during bacterial growth and are likely important for E. chaffeensis survival in its hostile host environments and that they may contribute to pathogenicity similar to other Gram negative bacteria (Du et al., 2005; Delory et al., 2006; Slamti et al., 2007; Matsui et al., 2008; Spector and Kenyon, 2012). For example, DnaK in Vibrio cholerae, the gene expression regulated by its $\sigma^{32}$, is involved in causing virulence in a host (Sahu et al., 1994; Chakrabarti et al., 1999; Slamti et al., 2007).

Prior research on E. coli (a $\gamma$-proteobacteria) provides abundant knowledge regarding gene regulation from its $\sigma^{32}$ and $\sigma^{70}$-bound RNAP, while such knowledge for both $\sigma^{32}$-and $\sigma^{70}$ bound RNAP in other Gram-negative bacteria, particularly for $\alpha$-proteobacteria, including for pathogenic organisms is very limited. Importantly, it is unclear how intracellular pathogens, such as E. chaffeensis, regulate gene expression to overcome the host stress and adapt to host environmental changes within its arthropod (tick) and vertebrate hosts. To extend knowledge on how E. chaffeensis regulates its gene expression, we continue investigations in defining the functions of its RNAP holoenzyme comprising $\sigma^{32}$ or $\sigma^{70}$. Transcription derived by an RNAP typically implicates in recognizing and binding to DNA sequence motifs of a promoter; -10 and -35 regions, and the spacer sequences located between the two motifs of a gene promoter (Gross et al., 1998; Paget and Helmann, 2003).

In the current study, we described the mapping of $d n a K$ gene promoter recognized primarily by the E. chaffeensis RNAP containing $\sigma^{32}$ using the previously developed $E$. coli surrogate system in the strain, CAG57101 (Koo et al., 2009a; Liu et al., 2013). In E. coli CAG57101, its endogenous rpoH gene (encoding for $\sigma^{32}$ ) is inactivated (Koo et al., 2009a) and in its place, we expressed the E. chaffeensis $\sigma^{32}$ from a plasmid in defining dnaK promoter mapping, as direct gene mapping studies are not possible in this and other related intracellular rickettsials. We also investigated the functional domains of E. chaffeensis $\sigma^{32}$ likely important for the RNAP function and 
in its interactions with the -10 motif and the spacer sequence of dnaK.

\section{MATERIALS AND METHODS}

\section{E. coli Strains and Plasmids}

Escherichia coli strains used in this study were TOP10 (Invitrogen Technologies, Carlsbad, CA, United States), BL21(DE3) (Novagen, San Diego, CA, United States), and CAG57101 (Koo et al., 2009a). Several plasmid constructs used in this study were obtained from commercial sources or modified from one or more of the existing plasmids. They include the derivatives of pSAKT-Eco_rpoH (previously known as pSAKT32) (Wang and deHaseth, 2003; Koo et al., 2009a), pQF50K-Ech_dnaK (Liu et al., 2013) and pMT504 (Tan and Engel, 1996). Genetic makeup of plasmids described in this study were included in Supplementary Table S1, except those obtained from a commercial source. The plasmid pSAKT-Eco_rpoH containing a p15A origin of replication and an ampicillin resistance gene has E. coli $r p o H$ gene under the control of IPTG inducible $\mathrm{P}_{\text {lac }}$ promoter (Wang and deHaseth, 2003; Koo et al., 2009a). The E. coli $r p o H$ from this plasmid was removed by using Q5 SiteDirected Mutagenesis Kit (New England Biolab, Inc., Ipswich, MA, United States) and the modified plasmid was referred as the pSAKT. The pSAKT-Ech_rpoH (previously known as pSAKT32-Ech_rpoH) contained E. chaffeensis rpoH (Liu et al., 2013). E. chaffeensis $r p o H$ variants with substitutions within the 2.3, 2.4, and 3.0 regions of $\sigma^{32}$ were constructed by mutagenesis using Q5 Site-Directed Mutagenesis Kit (New England Biolab, Inc., Ipswich, MA, United States). The names of the modified pSAKT-Ech_rpoH are provided in Supplementary Table S1.

The pQF50K-Ech_dnaK plasmid and the pQF50K-Ech_dnaK with deletion of -35 motif, which contains the $\beta$-galactosidase coding sequence (lacZ) with a pMB1 origin of replication and with a kanamycin resistance gene, was reported earlier (Liu et al., 2013). The modified plasmid with deletion of -10 motif was generated from pQF50K-Ech_dnaK plasmid using Q5 Site-Directed Mutagenesis Kit (New England Biolab, Inc., Ipswich, MA, United States). Site directed mutagenesis constructs with mutations at every nucleotide of the -10 motif were also generated from the pQF50KEch_dnaK plasmid using Q5 Site-Directed Mutagenesis Kit. The mutants to modify the AT rich spacer sequence of the dnaK promoter were generated similarly by modifying the pQF50K-Ech_dnaK plasmid. The expression plasmids of E. chaffeensis wildtype $\sigma^{32}$ was constructed and used for preparing purified recombinant proteins of $\sigma^{32}$ as in early reports (Liu et al., 2013).

For in vitro transcription analysis, pMT504-Ech_dnaK as transcription template was prepared and reported earlier (Liu et al., 2013). Constructs with various mutations at -10 motif for the dnaK promoter for in vitro transcription assays were similarly prepared from this plasmid using Q5 Site-Directed Mutagenesis Kit. The lengths of transcripts for the various promoter segments of $d n a K$ gene are 162 nucleotides. Integrity of all cloned segments in the plasmid constructs was confirmed by DNA sequence analysis using CEQ 8000 Genetic Analysis System (Beckman Coulter, Fullerton, CA, United States). The names of all engineered plasmids were listed in Supplementary Table S1. Mutagenic oligonucleotides were described in the Supplementary Table S2.

\section{E. coli Growth Conditions and $\beta$-Galactosidase Assays}

The E. coli strain CAG57101 transformed with the recombinant plasmids were grown as in early reports (Koo et al., 2009a; Liu et al., 2013). Briefly, cultures were grown at $30^{\circ} \mathrm{C}$ in LuriaBertani (LB) medium with chloramphenicol $(30 \mu \mathrm{g} / \mathrm{ml})$ and spectinomycin $(50 \mu \mathrm{g} / \mathrm{ml})$ in support of the strain's growth, and by ampicillin $(100 \mu \mathrm{g} / \mathrm{ml})$ for maintaining the pSAKTderived plasmids. To assess the functions and impact of various mutations within the promoter regions of genes encoding $d n a K$, pQF50K-derived plasmid containing the promoter segments were also maintained by growing E. coli cultures with the addition of kanamycin $(50 \mu \mathrm{g} / \mathrm{ml})$. E. coli cultures of CAG57101 in LB medium were grown overnight with appropriate antibiotic supplements which were diluted 1:100 into a fresh medium containing appropriate antibiotics and the growth was continued for $2 \mathrm{~h}$. Subsequently, cultures were then induced with $1 \mathrm{mM}$ IPTG for $3 \mathrm{~h}$ before harvesting, when OD at $600 \mathrm{~nm}$ reached between 0.6 and 0.8. Lysates were prepared and used to measure $\beta$-galactosidase enzyme activity using a $\beta$-Gal Assay Kit (Invitrogen Technologies, Carlsbad, CA, United States). All experiments were performed three independent times with independently grown cultures; specific activity of $\beta$-galactosidase was calculated as outlined in the kit protocol.

\section{In vitro Transcription Assays}

In vitro transcription reactions were performed in $10 \mu \mathrm{l}$ reaction mixture containing 0.1 picomoles each of the supercoiled plasmid DNA as the template and using RNAP holoenzyme containing recombinant E. chaffeensis $\sigma^{32}$ (Liu et al., 2013). The holoenzyme was prepared by mixing $0.5 \mu \mathrm{l}$ of 1:10 diluted stock of $E$. coli core enzyme (Epicentre, Madison, WI, United States) mixed with 10 -fold molar excess of purified recombinant E. chaffeensis $\sigma^{32}$ and kept in ice for $30 \mathrm{~min}$ prior to using for the reactions. The transcription reactions were performed at $37^{\circ} \mathrm{C}$ for $20 \mathrm{~min}$, and the reactions were terminated by adding $7 \mu \mathrm{l}$ of stop solution (95\% formamide, $20 \mathrm{mM}$ EDTA, 0.05\% bromophenol blue and $0.05 \%$ xylene cyanol). Six microliters each of the samples were resolved on a $6 \%$ polyacrylamide sequencing gel with $7 \mathrm{M}$ urea, then gels were transferred to a Whatman paper, dried and 162 nucleotide transcripts were visualized by exposing an $\mathrm{X}$-ray film to the gels. The transcripts were quantified using ImageJ software ${ }^{1}$.

\section{Bioinformatics}

Multiple DNA alignments were done using the programs Clustal X version 2.0 with default parameters (Larkin et al., 2007).

\footnotetext{
${ }^{1}$ http://rsb.info.nih.gov/ij
} 


\section{Statistical Analysis}

Statistical analyses were performed using Student's $t$-test, and a $P$-value $<0.05$ was considered significant with a single asterisk.

\section{RESULTS}

\section{The -10 Motif Is Needed for the E. chaffeensis dnak Gene Transcription by RNAP Holoenzyme Constituting of Its $\sigma^{32}$}

Our prior studies demonstrated that -35 motif, but not -10 motif, is required for the $\sigma^{70}$-bound RNAP holoenzyme transcription from $\sigma^{70}$-dependent promoters in E. chaffeensis (Liu et al., 2016). We also reported earlier that -35 motif is similarly required for the $d n a K$ gene transcription by E. chaffeensis $\sigma^{32}$-bound RNAP holoenzyme (Liu et al., 2013). To test whether or not the -10 motif of $d n a K$ promoter is required for the $\sigma^{32}$-dependent gene regulation, plasmid constructs lacking -10 or -35 motifs of the promoter were cloned upstream to the $\beta$-galactosidase coding sequence in an E. coli mutant deficient for its $\sigma^{32}$ expression that is functionally complemented with the E. chaffeensis $\sigma^{32}$ (Figure 1). The -10 motif deletion and similarly the -35 motif deletion resulted in a significant reduction of $\beta$-galactosidase activity (to 11 and $21 \%$, respectively; $p \leq 0.0005)$ compared to the dnaK wildtype (WT). The reduction of the $\beta$-galactosidase activity for the -10 motif deletion was similar to the negative control where the promoter segment was absent (NP).

\section{Identifying the Critical Sequence Determinants of -10 Motif of E. chaffeensis dnaK}

The consensus sequence of -35 motif for E. chaffeensis promoters recognized by its $\sigma^{32}$ (TTGTAT) is similar to the consensus

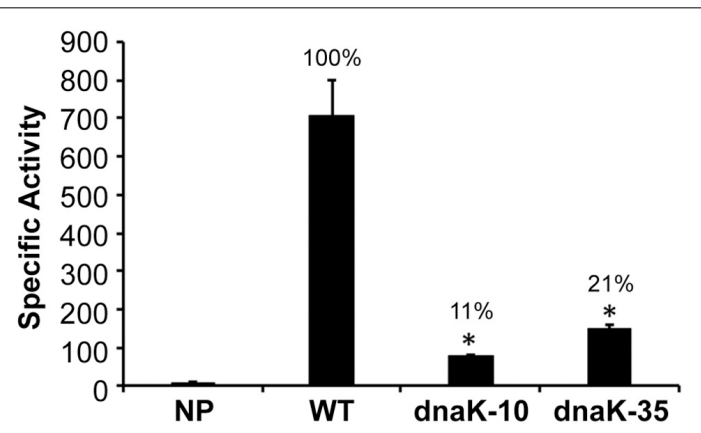

FIGURE 1 | Importance of -10 and -35 motifs of $E$. chaffeensis dnaK gene promoter assessed in E. coli CAG57101 expressing E. chaffeensis $\sigma^{32}$. The $\beta$-galactosidase expression driven by $E$. chaffeensis wild-type dnaK (WT) and the promoter with -35 motif or -10 motif deletions (dnaK-10 and dnaK-35) was assessed relative to no promoter (NP) control. Significant changes in the $\beta$-galactosidase were identified compared with the data observed for WT. All values are averages of at least three independent experiments; error bars indicated one standard deviation. ${ }^{*} p$-value $<0.05$.
-35 motif of $\sigma^{32}$-dependent promoters in E. coli (TTGAAA) and similarly it shares extensive homology to -35 motif for the genes recognized by $\sigma^{70}$ (TTGNTT) (Nonaka et al., 2006; Liu et al., 2013). The consensus -10 motif of E. chaffeensis promoters recognized by its $\sigma^{32}$ (TATATN) is also similar to its consensus -10 motif recognized by its $\sigma^{70}$ (TATTNT), however, it differs significantly from the consensus -10 motif of $E$. coli $\sigma^{32}$-dependent promoters (CCCCATWT) (Nonaka et al., 2006; Koo et al., 2009a; Liu et al., 2013). While deletion of -10 motif from $\sigma^{70}$-dependent promoters has no impact on promoter activities in E. chaffeensis (Liu et al., 2016), such deletion from the $\sigma^{32}$-dependent dnaK resulted in significant and $90 \%$ reduction in the promoter activity (Figure 1). These novel data suggest that, contrary to $\sigma^{70}$-bound RNAP (Liu et al., 2016), the -10 motif plays a critical role for $\sigma^{32}$-bound RNAP in E. chaffeensis. We therefore performed detailed point mutation experiments to define the critical sequence determinants of the -10 motif for the dnaK promoter activity (Figure 2 ). We have made substitution mutations at each base of the six-nucleotide motif (TATATC) and evaluated the impact of each mutation by measuring changes in $\beta$-galactosidase expression in CAG57101 E. coli functionally complemented with $E$. chaffeensis $\sigma^{32}$. A specific substitution mutation was indicated by combination of letters and numbers. For example, T1A indicates a change from $\mathrm{T}$ to $A$ transversion at the first position in the -10 motif. One or more substitutions at all six nucleotide positions resulted in significant decline in the promoter activity of $d n a K$. Substitutions at the first five nucleotides to any other nucleotide resulted in significant promoter activity drop. Substitutions in the first position from $\mathrm{T} 1 \mathrm{G}$ or $\mathrm{T} 1 \mathrm{C}$ resulted in the near complete loss of promoter activity, while T1A resulted in the reduction of promoter activity to $39 \%$. Similarly, in the second position, A2C and A2T mutations caused significant loss of promoter activity (reduced to $17 \%$ and $7 \%$ compared to the wildtype), whereas A2G mutation caused decline of two thirds of activity similar to T1A substitution. In the third and fourth positions, changes to any other nucleotide had the greatest impact in promoter activity decline (78-98\% reduction). Mutations in the fifth position to T5G or T5C had a greater impact (a near $90 \%$ decline), while reduction was less apparent for the $\mathrm{T} 5 \mathrm{~A}$ that is similar to the mutations in the first position $\mathrm{T}$. In the sixth position, only C6G mutation resulted in significant decline in the promoter activity to $35 \%$.

\section{In vitro Transcription Assays to Verify Sequence Determinants of -10 Motif Mapped in E. coli CAG57101}

To validate the results in defining the -10 motif in the E. coli surrogate system, we performed in vitro transcription assays using several randomly selected promoter mutation constructs; the assays were performed using RNAP holoenzyme reconstituted with the recombinant E. chaffeensis $\sigma^{32}$. We selected five $d n a K-10$ motif mutants for this experiment and compared the results with the wildtype promoter. Both wildtype and mutated versions of $d n a K$ promoter segments were cloned into the G-less cassette and used as templates in the in vitro 


\section{dnaK -10 motif (TATATC) point mutant}

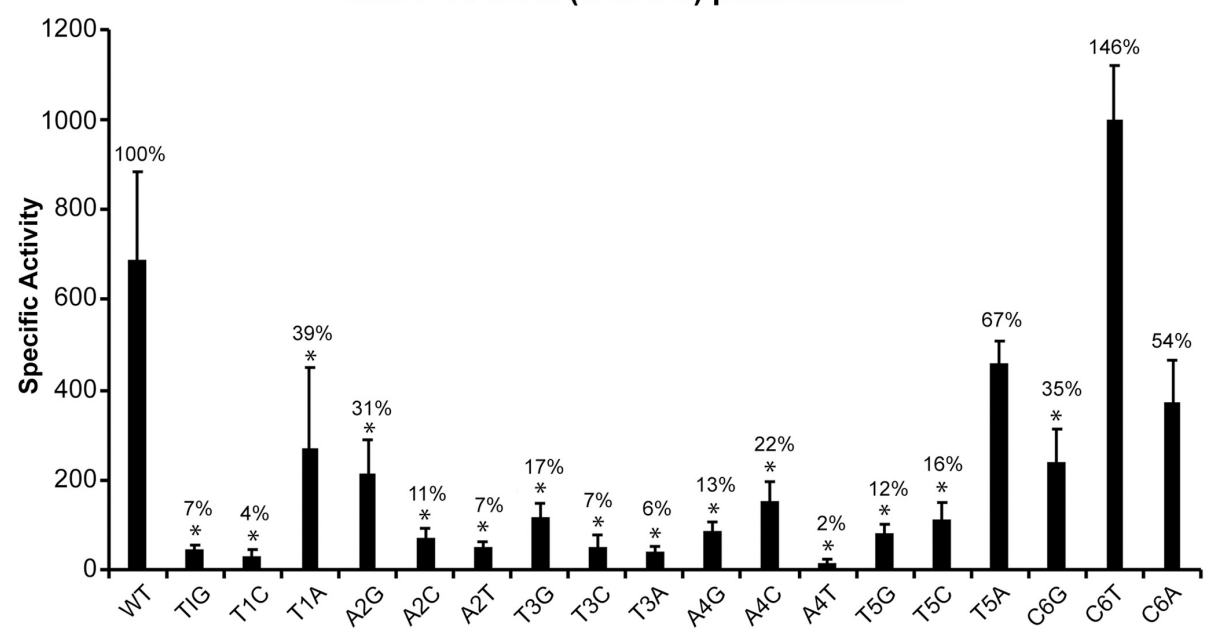

FIGURE 2 | Mapping the sequence determinants of -10 motifs in $E$. chaffeensis dnaK gene promoter. The $\beta$-galactosidase expression driven by E. chaffeensis promoter constructs containing point mutations at each of the six nucleotide positions of the -10 motifs of dnaK were measured in CAG57101 expressing E. chaffeensis $\sigma^{32}$. The experiment included the wildtype promoter control (WT). Each mutation is identified with a change of the nucleotide at each position to the modified nucleotide. $\beta$-galactosidase expression was presented relative to WT control. All values are averages of at least three independent experiments; error bars indicated one standard deviation. ${ }^{*} p$-value $<0.05$.

transcription assays (Figures 3A,B). Consistent with the results recorded with the E. coli CAG57101 system, mutants TIA, T1G and $\mathrm{A} 2 \mathrm{G}$ produced lesser transcripts compared to the wildtype $d n a K$. There was no significant difference for the T5A mutation compared to the wildtype, which is also consistent with the results observed in the E. coli system. In the sixth position, C6T mutation caused an increase of in vitro transcript level, which is also similar to the enhanced promoter activity observed in the E. coli CAG57101experiments.

\section{The Spacer Sequences Affect Promoter Activity}

Previous studies in E. coli demonstrate that spacer sequences located between -10 and -35 motifs contribute to promoter activities (Aoyama et al., 1983; Mulligan et al., 1985; HookBarnard and Hinton, 2007, 2009; Singh et al., 2011). In particular, nucleotides present in the spacer sequence and its length play critical role for a promoter activity. Further, a short C-rich region upstream to -10 motif in $E$. coli and in other $\gamma$-proteobacteria is identified as important extended -10 motif required for efficient transcription by $\sigma^{32}$-containing RNAP holoenzyme (Nonaka et al., 2006; Slamti et al., 2007; Koo et al., 2009a; Stoll et al., 2009), while such C-rich sequence does not exist in E. chaffeensis gene promoters, including in the $d n a K$ promoter. Our previous studies for $\sigma^{70}$-dependent promoter genes suggested that changes to the spacer sequence impact a promoter activity (Liu et al., 2016). We, therefore, investigated the importance of $d n a K$ spacer sequence, including in determining about how the lack of C-rich sequence impact the promoter activity. Nine spacer mutants were prepared where nucleotides within the spacer sequence were modified; they included replacing the spacer sequence with its complementary sequence (CP), or with a high GC content spacer

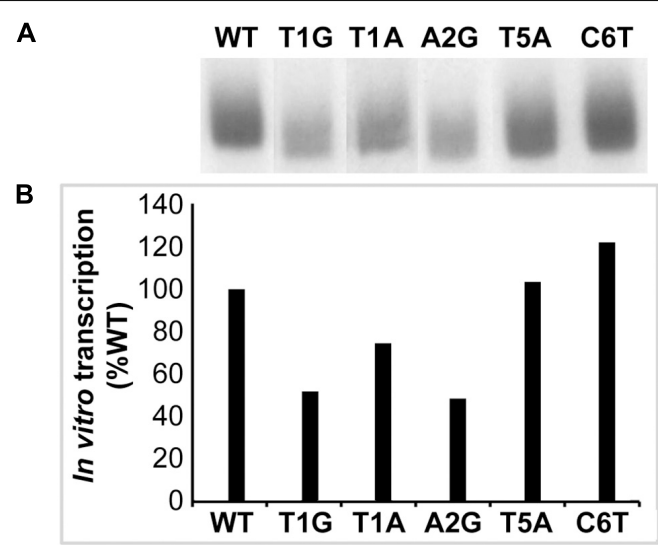

FIGURE $\mathbf{3}$ | In vitro transcription analysis validating the $E$. chaffeensis gene promoter mutants spanning the -10 motifs. Five randomly selected mutations at -10 motifs of $d n a K$ were examined by in vitro transcription assays using RNAP holoenzyme containing $E$. chaffeensis recombinant $\sigma^{32}$. The mutants of promoter segments cloned upstream to the G-less cassette in the pMT504 plasmid were used in the assays. The abundance of transcripts was captured as ${ }^{32} \mathrm{P}$ incorporation. Intensity of a band signals in a gel for in vitro transcripts made for the wild-type and mutant $d n a K$ were assessed using the software ImageJ (https://imagej.nih.gov/ij/). Panel (A) has the image data and panel (B) included the quantitative data collected from the image signals. The bars show the relative transcription products of mutant promoters as the percentage of transcripts compared to the wild-type promoter for E. chaffeensis recombinant $\sigma^{32}$. (WT and various mutant promoter constructs are identified as in Figure 2).

while keeping the spacer length constant (GC), or by increasing the spacer sequence lengths from $17 \mathrm{bp}$ to 18,19 , or $20 \mathrm{bps}$ or by decreasing it to 16,15 , or $14 \mathrm{bp}$ and finally by deleting the spacer sequence completely. These different spacer mutant constructs 
were depicted in Figure 4A. The $\beta$-galactosidase expression was then assessed for all these modified spacer promoter segments and compared to wildtype (WT) promoter construct in the E. coli surrogate system (CAG57101) (Figure 4B). The CP mutant caused a minor, non-significant increase in the promoter activity (30\% increase). The spacer substitution with GC resulted in a significant $50 \%$ decline in the $\beta$-galactosidase expression. Increasing the spacer length to $18 \mathrm{bp}$ or decreasing to 14 or 15 bp caused a major decline in the promoter activity, although the greatest decline was observed with the $14 \mathrm{bp}$ spacer (96\% drop), while reducing the length to 16 bp had no impact. Increasing the spacer lengths to 19 or 20 bp resulted in much higher enhancement of the promoter activity (586\% and $249 \%$, respectively). Deletion of the entire spacer sequence had no impact on the promoter activity compared to the WT promoter. We detected the presence of another $15 \mathrm{bp}$ spacer like sequence and an alternative -35 motif sequence in the complete deletion spacer construct; thus, it is highly likely that these sequences served as alternate spacer and -35 motif for the RNAP (Supplementary Figure S1).

\section{Substitution Mutations in E. chaffeensis $\sigma^{32}$}

Prior studies in E. coli revealed that the -10 motif of $\sigma^{32}$ dependent promoters is recognized by few amino acids within the regions 2.3, 2.4, and 3.0 of $\sigma^{32}$ protein (Kourennaia et al., 2005; Koo et al., 2009a). To determine what amino acids spanning these regions of $E$. chaffeensis $\sigma^{32}$ would contribute to the promoter activity, we have made substitution mutations at 6 different amino acid positions likely alter the functional domains of the pathogen $\sigma^{32}$. The amino acids for substitution mutations were selected based on their homology with the E. coli $\sigma^{32}$ (Supplementary Figure S2). Tryptophan (W) 108 of E. coli $\sigma^{32}$

\begin{tabular}{|c|c|c|c|}
\hline Name & -35 & Spacer & -10 \\
\hline WT & ITGTAA & TCTTATGATTTGGTTAT & TATATCTGTGATTA \\
\hline $\mathrm{CP}$ & ITGTAA & AGAATACTAAACCAATA & IATATCTGTGATTA \\
\hline GC & ITGTAA & TCCGAGGGTCCGGTTAT & IATATCTGTGATTA \\
\hline 20bp & ITGTAA & TCTTATGATT ata TGGTTAT & TATATCTGTGATTA \\
\hline $19 \mathrm{bp}$ & ITGTAA & TCTTATGATT a a TGGTTAT & TATATCTGTGATTA \\
\hline 18bp & ITGTAA & TCTTATGATT a TGGTTAT & TATATCTGTGATTA \\
\hline $16 \mathrm{bp}$ & ITGTAA & TCTTATGATT-GGTTAT & IATATCTGTGATTA \\
\hline $15 \mathrm{bp}$ & ITGTAA & TCTTATGAT--GGTTAT & IATATCTGTGATTA \\
\hline 14bp & ITGTAA & TCTTATGA---GGTTAT & TATATCTGTGATTA \\
\hline Obp & ITGTAA & - & TATATCTGTGATTA \\
\hline
\end{tabular}

B Activity of dnaK promoter with spacer mutant

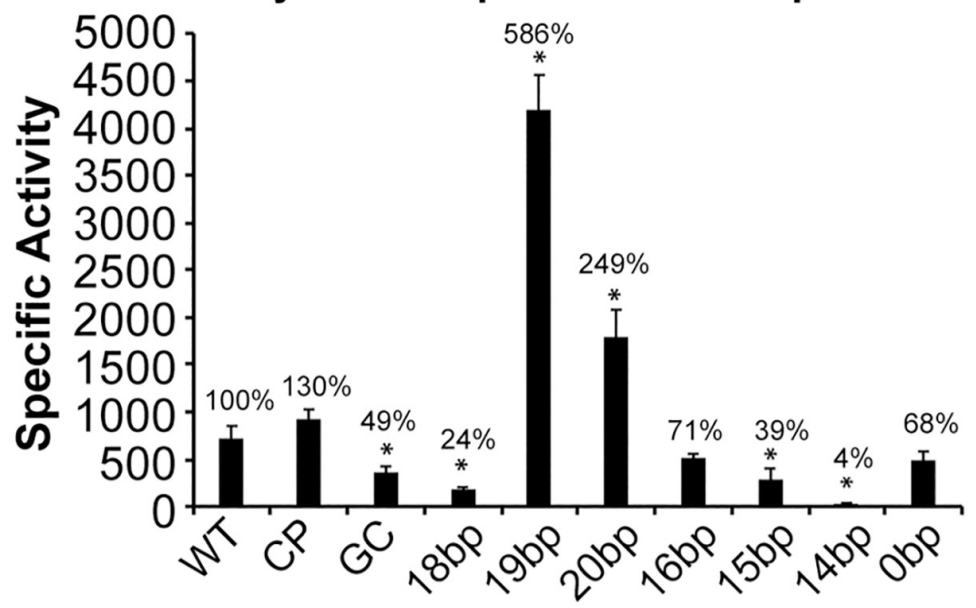

FIGURE 4 | AT-rich spacer sequence located between -10 and -35 motif contributes to altering the promoter activity of Ehrlichia chaffeensis dnaK gene. Promoter fragments used in the assays are as in Supplementary Figure $\mathbf{S 1}$ for wild-type dnaK. (A) Sequence spanning from +1 to -35 motif and the AT-rich spacer sequence is presented for the wild-type construct (WT) and for the constructs with modified spacer sequences which included replacing the AT-rich spacer with complementary sequence (CP), with GC rich spacer sequence (GC), changing the size of spacer from $17 \mathrm{bp}$ (WT) to $20 \mathrm{bp}$ (20bp), $19 \mathrm{bp}$ (19bp), $18 \mathrm{bp}$ (18bp), $16 \mathrm{bp}$ (16bp), $15 \mathrm{bp}$ (15bp), and $14 \mathrm{bp}$ (14bp) and deleting entire $17 \mathrm{bp}$ spacer (0bp). Lower case with underline indicated inserted nucleotides in spacer sequence and the break line refers to the deletion nucleotides from WT dnaK promoter. (B) The $\beta$-galactosidase activity was driven by promoters of WT, CP, GC, 20bp, 19bp, 18bp, 16bp, 15bp, 14bp, and 0bp in E. coli (CAG57101) with expressing E. chaffeensis $\sigma^{32}$. All values are averages of at least three independent experiments; error bars indicated one standard deviation. ${ }^{*} p$-value $<0.05$. 
within the region 2.4 is identified as important for recognition of $-13 \mathrm{C}$ in a promoter recognized by it (Kourennaia et al., 2005; Koo et al., 2009a). In E. chaffeensis $\sigma^{32}, \mathrm{~W}$ is located at 106. Glutamic acid (E) at position 112 in E. coli $\sigma^{32}$ is also implicated for its contribution to $-13 \mathrm{C}$ recognition and interaction with its $\sigma^{32}$-dependent promoters (Koo et al., 2009a). Phenylalanine (F) at position 110 of E. chaffeensis $\sigma^{32}$ is in the homologous position to $\mathrm{E} 112$ in E. coli $\sigma^{32}$. As E. chaffeensis dnaK lacks C-rich region upstream to its -10 motif, we expected that amino acid substitution mutations at these two positions in E. chaffeensis $\sigma^{32}$ would not have any impact. Lysine $(\mathrm{K})$ at position 130 in region 3.0 of $E$. coli $\sigma^{32}$ is similarly implicated for the recognition of the C-rich region for the $\sigma^{32}$-promoters; groE and $\operatorname{grpE}$ (Koo et al., 2009a). Glutamin (Q) at position 128 in region 3.0 of E. chaffeensis $\sigma^{32}$ is the amino acid at the homologous position to K130 of E. coli $\sigma^{32}$. Substitution of W106 to A caused significant reduction of the $\beta$-galactosidase expression $(80 \%$ decline), while F110 substitution to A or E (alanine or glutamic acid) in E. chaffeensis $\sigma^{32}$ did not significantly alter the promoter function (Figure 5). Similarly, substitution of Q128 to A did not significantly impact $E$. chaffeensis dnaK promoter activity. The mutational data with the exception of W106 are consistent with the lack of C-rich region in E. chaffeensis. However, W106 may be critical for the promoter activity independent of the C-rich sequence, at least in E. chaffeensis. Phenylalanine (F) at position 104 within the 2.3 region of $E$. coli $\sigma^{32}$ is identified as critical for its structural integrity and activity of $\sigma^{32}$ (Kourennaia et al., 2005). In E. chaffeensis $\sigma^{32}$, a polar amino acid (at position 102) \{tyrosine (Y)\} is present at the position homologous to F104. Similarly, A111 of E. coli $\sigma^{32}$ is implicated for its binding to core RNAP (Kourennaia et al., 2005). Y102 to A caused a significant decline of $E$. chaffeensis $\sigma^{32}$ activity ( $88 \%$ reduction). Likewise, substitution of A109 to glutamine (Q) in E. chaffeensis $\sigma^{32}$ that is homologous to A111 of E. coli $\sigma^{32}$ resulted in a significant decline of activity (about $31 \%$ of WT level). Previous studies in E. coli demonstrate that substitution of F136A within the region 3.0 reduces the interaction between core RNAP and $\sigma^{32}$ thus leading to $80 \%$ decline in promoter activity for its groE gene (Joo et al., 1997; Kourennaia et al., 2005; Koo et al., 2009a). A similar substitution in E. chaffeensis $\sigma^{32}$; F134A also caused similar decline of its activity for the $d n a K$ promoter (reduced to $28 \%$ activity compared to WT E. chaffeensis $\sigma^{32}$ ). Together, E. chaffeensis $\sigma^{32}$ substitution mutation experiments allowed the identification of critical functional domains engaged in $\sigma^{32}$ bound RNAP interactions with the $d n a K$ promoter, including in confirming that the C-rich region is not critical for its function.

\section{Differences in the -10 Motifs Between $E$. coli and E. chaffeensis Are Sufficient in Having Differential $\sigma^{32}$ Functions}

Unlike $E$. coli and other $\gamma$ - proteobacterial gene promoters, -10 motif of $E$. chaffeensis $\sigma^{32}$-dependent dnaK promoter (TATATN) is distinct in lacking a C-rich sequence upstream to -10 motif (Nonaka et al., 2006; Koo et al., 2009a; Liu et al., 2013). Results presented in the previous section suggest that the $\mathrm{C}$-rich region is indeed not required for E. chaffeensis $\sigma^{32}$

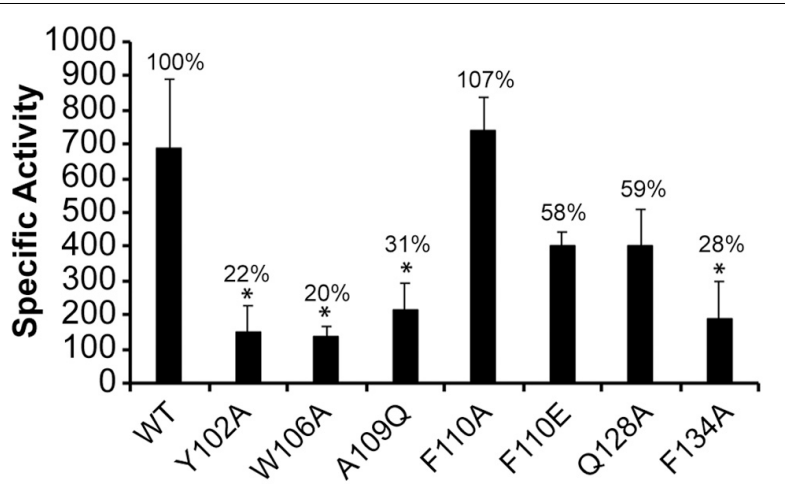

FIGURE 5 | Substitutions at six amino acids located in regions 2.3, 2.4, and 3.0 of $E$. chaffeensis $\sigma^{32}$ impacting the promoter activity of the wild-type E. chaffeensis dnaK. Mutations at six residues (Y102, W106, A109, F110, $\mathrm{Q} 128$, and $\mathrm{F} 134)$ of $E$. chaffeensis $\sigma^{32}$ were assessed with the wild-type dnaK promoter. $\beta$-galactosidase expression was measured for the mutant proteins relative to the wild-type (WT) E. chaffeensis $\sigma^{32}$ in CAG57101. All values are averages of at least three independent experiments; error bars indicated one standard deviation. ${ }^{*} p$-value $<0.05$.

dependent $d n a K$ promoter function. To further map how the variations in -10 motif and spacer sequence in E. chaffeensis dnaK make it unique for this intracellular pathogen promoter function, we prepared two modified constructs where two or four nucleotides spanning between the spacer sequence and -10 motif (TT or TATT, respectively) were replaced with either two Cs or four Cs (Figure 6A). Importantly, these sequence modifications change the E. chaffeensis -10 motif to be more similar to the E. coli consensus -10 motif (CCCCATWT) (Figure 6A). The modified constructs having $2 \mathrm{Cs}$ and $4 \mathrm{Cs}$ were then assessed in the E. coli surrogate system expressing either E. chaffeensis $\sigma^{32}$ (Ech- $\left.\sigma^{32}\right)$ or E. coli $\sigma^{32}\left(\right.$ Eco- $\left.\sigma^{32}\right)$. Compared to the wildtype dnaK promoter, both the $2 \mathrm{C}$ and $4 \mathrm{C}$ mutants caused drastic decline in the $\beta$-galactosidase expression by as much as 15 -fold for E. chaffeensis $\sigma^{32}$ (Figure 6B). On the contrary, while wildtype $d n a K$ promoter had a minimal $\beta$-galactosidase expression with the E. coli $\sigma^{32}$, the $2 \mathrm{Cs}$ and $4 \mathrm{Cs}$ substitutions caused a significant and a step-wise increase of $\beta$-galactosidase expression to 2.8fold and 14.5-fold, respectively. Together, these results suggest that the inclusion of $\mathrm{C}$-rich sequences is sufficient in altering the promoter specificities of E. chaffeensis to be similar to E. coli $\sigma^{32}$ dependent RNAP.

\section{DISCUSSION}

Because obligate intracellular bacteria reside within the cytosol or a phagosome of an infected host cell, they encounter minimal environmental changes, possibly compared to freeliving bacteria, such as E. coli. Obligate intracellular bacteria typically have reduced genomes and consequently their genomes also have limited numbers of sigma factors. For example, E. chaffeensis genome has only two sigma factor genes encoding for a constitutive and an alternative sigma factor, $\sigma^{70}$ and $\sigma^{32}$, respectively (Dunning Hotopp et al., 2006). On the contrary, a 
A

\begin{tabular}{|l|lr|}
\hline & \multicolumn{1}{|c}{ spacer } & -10 \\
\hline dnaK (WT) & TTGTAATCTTATGATTTGGTTAT TATATCTGTGATTA \\
dnaK (2C) & ITGTAATCTTATGATTTGGTTACCATATCTGTGATTA \\
dnaK (4C) & $\underline{\text { ITGTAATCTTATGATTTGGTCCCCATATCTGTGATTA }}$ \\
E. coli & TTGAAA & CCCCATNT \\
\hline
\end{tabular}

B

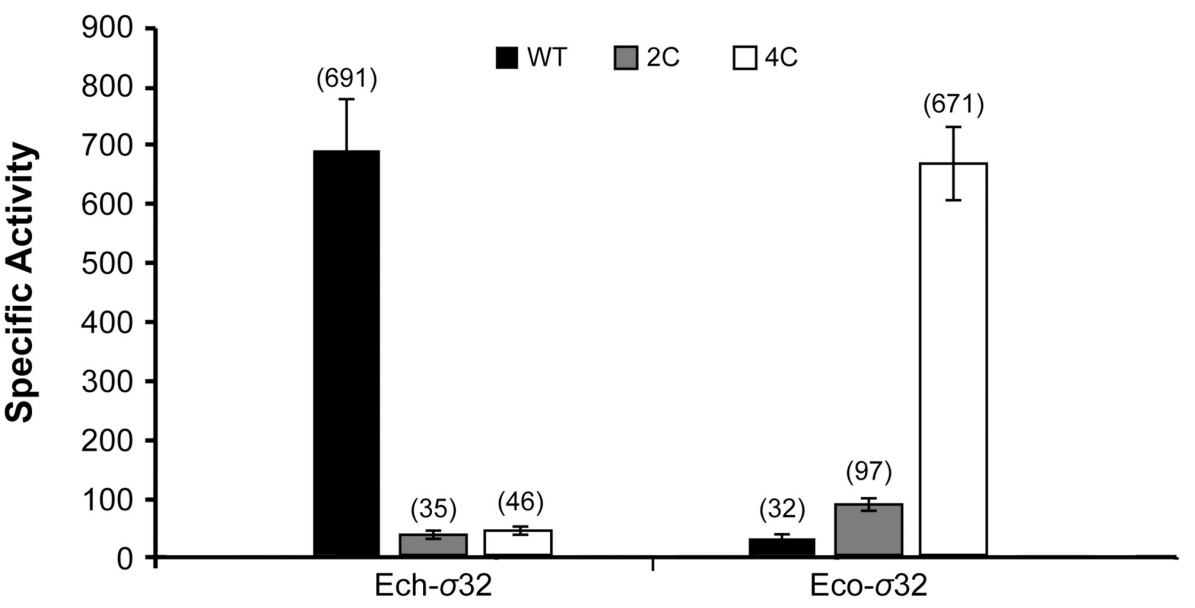

FIGURE 6 | Sequence variations in -10 motif and its immediate upstream sequence define specificities for $E$. coli- and $E$. chaffeensis $\sigma^{32}$. The -10 motif for wild-type dnaK promoter (WT) and for the modified sequences where TT and TATT near -10 motif are modified to CC (2C) and CCCC (4C), respectively (panel A). The inclusion of $2 \mathrm{C}$ and $4 \mathrm{C}$ make the -10 motif of dnaK promoter to be similar to consensus sequence of -10 motif for $E$. coli. E. chaffeensis $\sigma^{32}$ (Ech- $\sigma^{32}$ ) and E. coli $\sigma^{32}\left(E c o-\sigma^{32}\right.$ ) recognized the WT, $2 \mathrm{C}$, and $4 \mathrm{C}$ promoters differently (panel B). $\beta$-galactosidase expression as specific activity values were in parenthesis. All values are averages of at least three independent experiments; error bars indicated one standard deviation.

non-pathogenic bacterium, such as E. coli, contains seven sigma factors, including $\sigma^{70}$ and $\sigma^{32}$ (Gruber and Gross, 2003; Tripathi et al., 2014). Alternate sigma factor; $\sigma^{32}$ is known to regulate genes involved in overcoming environmental stresses (Zhao et al., 2005; Martínez-Salazar et al., 2009). Prior studies in E. coli, V. cholerae, Neisseria gonorrhoeae, and Francisella tularensis suggest that $\sigma^{32}$ regulon involves several heat-shock proteins, also known as chaperon proteins; Hsp40, HspG, Dnak, DnaJ, GroES, and GroEL. These proteins are involved in temperatureinduced stress control of the organisms (Zhao et al., 2005; Gunesekere et al., 2006; Nonaka et al., 2006; Slamti et al., 2007). These proteins play critical roles in both preventing denaturation of proteins and also to promote renaturation of aggregated proteins (Gragerov et al., 1992; Nishihara et al., 1998; Mogk et al., 1999). Some of the proteins involved in regulating stress response, such as $\mathrm{ClpB}$, HspG, and DnaK, are also considered important for the intracellular survival of a pathogenic bacterium, F. tularensis (Tempel et al., 2006; Su et al., 2007; Weiss et al., 2007; Meibom et al., 2008). The sigma factor, $\sigma^{32}$, is identified as critical for promoting transcription of genes engaged in overcoming stressful environments for bacteria to promote the reduction of accumulation of misfolded and aggregated proteins (Gragerov et al., 1992; Slamti et al., 2007; Guisbert et al., 2008).
Ehrlichia chaffeensis and related Anaplasmataceae family pathogens have the ability adapt to vertebrate and tick hosts. However, it is unclear how tick transmitted pathogens regulate gene expression in tick and vertebrate hosts. Studying gene regulation will aid in understanding how rickettsial pathogens adapt to dual hosts and sense nutrient, starving, temperature, and other stressful environments within an infected host cell. Previous studies revealed that E. chaffeensis has two morphological forms, dense core cell as the pathogen's infectious form and reticulate cell, which replicates within a phagosome of an infected host cell (Zhang et al., 2007; Dedonder et al., 2012). We recently reported that a stress response protein, $\mathrm{ClpB}$, transcripts are higher during the replicative stage of the pathogen, while gene expression of another heat shock protein, DnaK, and the stress response sigma factor, RpoH, remained as constitutively expressed throughout the replicating stage (Liu et al., 2013; Zhang et al., 2013). Further, we also reported that E. chaffeensis sigma factors, $\sigma^{32}$ and $\sigma^{70}$, function cooperatively in transcribing pathogen genes, but with varying affinities (Liu et al., 2013). The dnaK has higher affinity for RNAP containing E. chaffeensis $\sigma^{32}$ compared to $\sigma^{70}$ (Liu et al., 2013). The current study is the first in defining E. chaffeensis $\sigma^{32}$-dependent gene promoter region of $d n a K$. 
Despite our recent advances in establishing tractable genetics for E. chaffeensis (Wang et al., 2017), studying gene regulation remains a challenge due to lack of a well-established methods to maintain extracellular plasmids, as the pathogen and other related Anaplasmataceae pathogens lack natural plasmids. To overcome this challenge, in the current study, we utilized the $E$. coli surrogate system to map promoter-binding domains required for gene expression in $E$. chaffeensis for a $\sigma^{32}$-dependent gene promoter. We validated the results from the E. coli surrogate system using in vitro transcription assays which we developed earlier (Liu et al., 2013, 2016). In vitro transcription system is applied well in understanding intracellular bacterial gene regulation for pathogens, such as Chlamydia trachomatis, for which a tractable genetic system is equally not well developed, which can support gene regulation studies (Mathews et al., 1993; Tan and Engel, 1996; Tan et al., 1998; Yu and Tan, 2003; Shen et al., 2004; Akers and Tan, 2006; Rao et al., 2009; Bao et al., 2011, 2012). Hence, in view of the technical challenges, methods described in the current study are innovative in mapping E. chaffeensis gene regulation, and that the study will lead the way for similar investigations in other related Anaplasmataceae family pathogens.

RNA polymerase holoenzyme containing $\sigma^{70}$ of $E$. coli transcribes housekeeping genes by recognizing two highly conserved motifs of a gene promoter; referred as -10 and -35 motifs (Gross et al., 1998). We reported earlier that the consensus -10 and -35 motifs for E. chaffeensis $\sigma^{70}$ dependent promoters (TATTNT and TTGNTT, respectively) (Liu et al., 2013) are similar to E. coli consensus -10 and -35 (TATAAT and TTGACA, respectively) (Singh et al., 2011; Shimada et al., 2014). We reported that the -35 motif and the AT-rich spacer sequences are important for a genespecific regulation by $\sigma^{70}$-dependent promoters (Liu et al., 2016). The predicted E. chaffeensis consensus -35 motif for the alternative sigma factor $\left(\sigma^{32}\right)$ is also similar to E. coli consensus -35 motif (Nonaka et al., 2006; Koo et al., 2009a,b; Liu et al., 2013). The -10 motif of E. chaffeensis $\sigma^{32}$-dependent promoters (TATATN), however, differs substantially from the consensus E. coli-10 motif (CCCCATNT) (Nonaka et al., 2006; Koo et al., 2009a,b; Liu et al., 2013). The consensus -35 motif is also extensively conserved among $\sigma^{32}$-dependent promoters in proteobacteria (Supplementary Table S3), while -10 motif of $\sigma^{32}$-dependent promoters vary considerably among various classes of proteobacteria. For example, many $\gamma$-proteobacteria contain a C-rich sequence upstream to the -10 motif and is implicated in regulating $\sigma^{32}$-dependent gene regulation (Nonaka et al., 2006; Slamti et al., 2007; Grall et al., 2009; Stoll et al., 2009). Such sequence, however, is absent in E. chaffeensis $\sigma^{32}$-dependent gene promoters (Liu et al., 2013). Thus, we hypothesized that the pathogen is evolved to possess a distinctive -10 motif and that it plays a critical role in $\sigma^{32}$-dependent promoter regulation. Previous studies suggest that the length of a spacer sequence between the -10 and -35 motifs and the composition of specific nucleotides present within a spacer affect promoter activity (Aoyama et al., 1983; Mulligan et al., 1985; Hook-Barnard and Hinton, 2009; Singh et al., 2011; Liu et al., 2016). In particular, spacer sequences influence the three-dimensional structure of a promoter and any modifications to a spacer sequence, therefore, affect the efficiency of interactions between the RNAP holoenzyme with a promoter sequence either positively or negatively (Rud et al., 2006; Hook-Barnard and Hinton, 2009; Singh et al., 2011). Consistent with prior studies, our current investigation similarly revealed the importance of spacer sequence in contributing to the promoter activity for the E. chaffeensis dnaK gene transcribed by its $\sigma^{32}$. We previously reported that modifications to spacer sequences similarly impact E. chaffeensis genes transcribed by $\sigma^{70}$ genes and that changes to spacer sequences alter the curvature of a promoter region (Liu et al., 2016).

In summary, we mapped E. chaffeensis dnaK-10 and -35 motifs and the spacer sequence upstream to it by performing detailed mutational analysis. Furthermore, this study confirmed that the $\mathrm{C}$-rich region-specific interactions between a gene promoter and $\sigma^{32}$ protein domains, known to be critical for E. coli, are absent in E. chaffeensis, which makes the pathogen gene regulation distinct, as judged from characterizing the dnaK gene promoter.

\section{DATA AVAILABILITY}

The datasets generated for this study are available on request to the corresponding author.

\section{AUTHOR CONTRIBUTIONS}

RG conceived and directed the research design. HL contributed to the research plan and performed the experiments. HL and RG evaluated the data and prepared the manuscript.

\section{FUNDING}

This work was supported by the PHS grant \# AI070908 from the National Institute of Allergy and Infectious Diseases, National Institutes of Health, United States. This manuscript is a contribution from the Kansas Agricultural Experiment Station (Contribution Number 19-291-J). The funders had no role in study design, data collection and interpretation, or the decision to submit the work for publication.

\section{ACKNOWLEDGMENTS}

We thank Ms. Mal Rooks Hoover for her help in preparing the figures.

\section{SUPPLEMENTARY MATERIAL}

The Supplementary Material for this article can be found online at: https://www.frontiersin.org/articles/10.3389/fmicb.2019. 01772/full\#supplementary-material 


\section{REFERENCES}

Akers, J. C., and Tan, M. (2006). Molecular mechanism of tryptophan-dependent transcriptional regulation in Chlamydia trachomatis. J. Bacteriol. 188, 4236-4243. doi: 10.1128/jb.01660-05

Aoyama, T., Takanami, M., Ohtsuka, E., Taniyama, Y., Marumoto, R., Sato, H., et al. (1983). Essential structure of E. coli promoter: effect of spacer length between the two consensus sequences on promoter function. Nucleic Acids Res. 11, 5855-5864. doi: 10.1093/nar/11.17.5855

Bao, X., Nickels, B. E., and Fan, H. (2012). Chlamydia trachomatis protein GrgA activates transcription by contacting the nonconserved region of sigma66. Proc. Natl. Acad. Sci. U.S.A. 109, 16870-16875. doi: 10.1073/pnas.1207300109

Bao, X., Pachikara, N. D., Oey, C. B., Balakrishnan, A., Westblade, L. F., Tan, M., et al. (2011). Non-coding nucleotides and amino acids near the active site regulate peptide deformylase expression and inhibitor susceptibility in Chlamydia trachomatis. Microbiology 157, 2569-2581. doi: 10.1099/mic.0. 049668-0

Breitschwerdt, E. B., Hegarty, B. C., and Hancock, S. I. (1998). Sequential evaluation of dogs naturally infected with Ehrlichia canis, Ehrlichia chaffeensis, Ehrlichia equi, Ehrlichia ewingii, or Bartonella vinsonii. J. Clin. Microbiol. 36, 2645-2651.

Browning, D. F., and Busby, S. J. W. (2016). Local and global regulation of transcription initiation in bacteria. Nat. Rev. Microbiol. 14:638. doi: 10.1038/ nrmicro.2016.103

Chakrabarti, S., Sengupta, N., and Chowdhury, R. (1999). Role of DnaK in in vitro and in vivo expression of virulence factors of Vibrio cholerae. Infect Immun. 67, 1025-1033.

Chamberlin, M., Kingston, R., Gilman, M., Wiggs, J., and Devera, A. (1983). Isolation of bacterial and bacteriophage RNA polymerases and their use in synthesis of RNA in vitro. Methods Enzymol. 101, 540-568. doi: 10.1016/00766879(83)01037-x

Cheng, C., Nair, A. D., Indukuri, V. V., Gong, S., Felsheim, R. F., Jaworski, D., et al. (2013). Targeted and random mutagenesis of Ehrlichia chaffeensis for the identification of genes required for in vivo infection. PLoS Pathog. 9:e1003171. doi: 10.1371/journal.ppat.1003171

Crosby, F. L., Wamsley, H. L., Pate, M. G., Lundgren, A. M., Noh, S. M., Munderloh, U. G., et al. (2014). Knockout of an outer membrane protein operon of Anaplasma marginale by transposon mutagenesis. BMC Genomics 15:278. doi: 10.1186/1471-2164-15-278

Darby, A. C., Cho, N.-H., Fuxelius, H.-H., Westberg, J., and Andersson, S. G. E. (2007). Intracellular pathogens go extreme: genome evolution in the Rickettsiales. Trends Genet. 23, 511-520. doi: 10.1016/j.tig.2007.08.002

Davidson, W. R., Lockhart, J. M., Stallknecht, D. E., Howerth, E. W., Dawson, J. E., and Rechav, Y. (2001). Persistent Ehrlichia chaffeensis infection in white-tailed deer. J. Wildl. Dis. 37, 538-546.

Dawson, J. E., Biggie, K. L., Warner, C. K., Cookson, K., Jenkins, S., Levine, J. F., et al. (1996). Polymerase chain reaction evidence of Ehrlichia chaffeensis, an etiologic agent of human monocytic ehrlichiosis, in dogs, from southeast Virginia. Am. J. Vet. Res. 57, 1175-1179.

Dedonder, S. E., Cheng, C., Willard, L. H., Boyle, D. L., and Ganta, R. R. (2012). Transmission electron microscopy reveals distinct macrophage- and tick cellspecific morphological stages of Ehrlichia chaffeensis. PLoS One 7:e36749. doi: 10.1371/journal.pone.0036749

Delory, M., Hallez, R., Letesson, J. J., and De Bolle, X. (2006). An RpoH-like heat shock sigma factor is involved in stress response and virulence in Brucella melitensis 16M. J. Bacteriol. 188, 7707-7710. doi: 10.1128/jb.00644-06

Du, Y., Lenz, J., and Arvidson, C. G. (2005). Global gene expression and the role of sigma factors in Neisseria gonorrhoeae in interactions with epithelial cells. Infect. Immun. 73, 4834-4845. doi: 10.1128/iai.73.8.4834-4845.2005

Dugan, V. G., Little, S. E., Stallknecht, D. E., and Beall, A. D. (2000). Natural infection of domestic goats with Ehrlichia chaffeensis. J. Clin. Microbiol. 38, 448-449.

Dumler, J. S., Sutker, W. L., and Walker, D. H. (1993). Persistent infection with Ehrlichia chaffeensis. Clin. Infect. Dis. 17, 903-905. doi: 10.1093/clinids/17.5.903

Dunning Hotopp, J. C., Lin, M., Madupu, R., Crabtree, J., Angiuoli, S. V., Eisen, J., et al. (2006). Comparative genomics of emerging human ehrlichiosis agents. PLoS Genet. 2:e21. doi: 10.1371/journal.pgen.0020021

Faburay, B., Liu, H., Peddireddi, L., and Ganta, R. R. (2011). Isolation and characterization of Ehrlichia chaffeensis RNA polymerase and its use in evaluating p28 outer membrane protein gene promoters. BMC Microbiol. 11:83. doi: 10.1186/1471-2180-11-83

Felsheim, R. F., Herron, M. J., Nelson, C. M., Burkhardt, N. Y., Barbet, A. F., Kurtti, T. J., et al. (2006). Transformation of Anaplasma phagocytophilum. BMC Biotechnol. 6:42. doi: 10.1186/1472-6750-6-42

Gragerov, A., Nudler, E., Komissarova, N., Gaitanaris, G. A., Gottesman, M. E., and Nikiforov, V. (1992). Cooperation of GroEL/GroES and DnaK/DnaJ heat shock proteins in preventing protein misfolding in Escherichia coli. Proc. Natl. Acad. Sci. U.S.A. 89, 10341-10344. doi: 10.1073/pnas.89.21.10341

Grall, N., Livny, J., Waldor, M., Barel, M., Charbit, A., and Meibom, K. L. (2009). Pivotal role of the Francisella tularensis heat-shock sigma factor RpoH. Microbiology 155, 2560-2572. doi: 10.1099/mic.0.029058-0

Gross, C. A., Chan, C., Dombroski, A., Gruber, T., Sharp, M., Tupy, J., et al. (1998). The functional and regulatory roles of sigma factors in transcription. Cold Spring Harb. Symp. Quant. Biol. 63, 141-155.

Gruber, T. M., and Gross, C. A. (2003). Multiple sigma subunits and the partitioning of bacterial transcription space. Annu. Rev. Microbiol. 57, 441-466. doi: 10.1146/annurev.micro.57.030502.090913

Guisbert, E., Yura, T., Rhodius, V. A., and Gross, C. A. (2008). Convergence of molecular, modeling, and systems approaches for an understanding of the Escherichia coli heat shock response. Microbiol. Mol. Biol. Rev. 72, 545-554. doi: 10.1128/MMBR.00007-08

Gunesekere, I. C., Kahler, C. M., Powell, D. R., Snyder, L. A. S., Saunders, N. J., Rood, J. I, et al. (2006). Comparison of the RpoH-dependent regulon and general stress response in Neisseria gonorrhoeae. J. Bacteriol. 188, 4769-4776. doi: 10.1128/jb.01807-05

Han, K., Li, Z. F., Peng, R., Zhu, L. P., Zhou, T., Wang, L. G., et al. (2013). Extraordinary expansion of a Sorangium cellulosum genome from an alkaline milieu. Sci. Rep. 3:2101. doi: 10.1038/srep02101

Hook-Barnard, I. G., and Hinton, D. M. (2007). Transcription initiation by mix and match elements: flexibility for polymerase binding to bacterial promoters. Gene Regul. Syst. Biol. 1, 275-293.

Hook-Barnard, I. G., and Hinton, D. M. (2009). The promoter spacer influences transcription initiation via $\sigma 70$ region 1.1 of Escherichia coli RNA polymerase. Proc. Natl. Acad. Sci. U.S.A. 106, 737-742. doi: 10.1073/pnas.0808133106

Ismail, N., Bloch, K. C., and Mcbride, J. W. (2010). Human ehrlichiosis and anaplasmosis. Clin. Lab. Med. 30, 261-292. doi: 10.1016/j.cll.2009.10.004

Joo, D. M., Ng, N., and Calendar, R. (1997). A $\sigma 32$ mutant with a single amino acid change in the highly conserved region 2.2 exhibits reduced core RNA polymerase affinity. Proc. Natl. Acad. Sci. U.S.A. 94, 4907-4912. doi: 10.1073/ pnas.94.10.4907

Kill, K., Binnewies, T. T., Sicheritz-Pontén, T., Willenbrock, H., Hallin, P. F., Wassenaar, T. M., et al. (2005). Genome update: sigma factors in 240 bacterial genomes. Microbiology 151, 3147-3150. doi: 10.1099/mic.0.28339-0

Kocan, A. A., Levesque, G. C., Whitworth, L. C., Murphy, G. L., Ewing, S. A., and Barker, R. W. (2000). Naturally occurring Ehrlichia chaffeensis infection in coyotes from Oklahoma. Emerg Infect. Dis. 6, 477-480. doi: 10.3201/eid0605. 000505

Koo, B. M., Rhodius, V. A., Campbell, E. A., and Gross, C. A. (2009a). Dissection of recognition determinants of Escherichia coli sigma32 suggests a composite -10 region with an 'extended -10' motif and a core -10 element. Mol. Microbiol. 72, 815-829. doi: 10.1111/j.1365-2958.2009.06690.x

Koo, B. M., Rhodius, V. A., Nonaka, G., Dehaseth, P. L., and Gross, C. A. (2009b). Reduced capacity of alternative $\sigma$ s to melt promoters ensures stringent promoter recognition. Genes Dev. 23, 2426-2436. doi: 10.1101/gad.184 3709

Kourennaia, O. V., Tsujikawa, L., and Dehaseth, P. L. (2005). Mutational analysis of Escherichia coli heat shock transcription factor sigma 32 reveals similarities with sigma 70 in recognition of the -35 promoter element and differences in promoter DNA melting and -10 recognition. J. Bacteriol. 187, 6762-6769. doi: 10.1128/jb.187.19.6762-6769.2005

Kuriakose, J. A., Miyashiro, S., Luo, T., Zhu, B., and Mcbride, J. W. (2011). Ehrlichia chaffeensis transcriptome in mammalian and arthropod hosts reveals differential gene expression and post transcriptional regulation. PLoS One 6:e24136. doi: 10.1371/journal.pone.0024136

Larkin, M. A., Blackshields, G., Brown, N. P., Chenna, R., Mcgettigan, P. A., Mcwilliam, H., et al. (2007). Clustal W and Clustal X version 2.0. Bioinformatics 23, 2947-2948. doi: 10.1093/bioinformatics/btm 404 
Liu, H., Jakkula, L. U. M. R., Von Ohlen, T., and Ganta, R. R. (2016). Sequence determinants spanning -35 motif and AT-rich spacer region impacting Ehrlichia chaffeensis Sigma 70-dependent promoter activity of two differentially expressed p28 outer membrane protein genes. DNA Res. 23, 495-505. doi: 10.1093/dnares/dsw034

Liu, H., Von Ohlen, T., Cheng, C., Faburay, B., and Ganta, R. R. (2013). Transcription of Ehrlichia chaffeensis genes is accomplished by RNA polymerase holoenzyme containing either sigma 32 or sigma 70. PLoS One 8:e81780. doi: 10.1371/journal.pone.0081780

Lockhart, J. M., Davidson, W. R., Stallknecht, D. E., Dawson, J. E., and Little, S. E. (1997). Natural history of Ehrlichia chaffeensis (Rickettsiales: Ehrlichieae) in the piedmont physiographic province of Georgia. J. Parasitol. 83, 887-894.

Long, S. W., Whitworth, T. J., Walker, D. H., and Yu, X. (2005). Overcoming barriers to the transformation of the genus Ehrlichia. Ann. N. Y. Acad. Sci. 1063, 403-410. doi: 10.1196/annals.1355.072

Martínez-Salazar, J. M., Sandoval-Calderón, M., Guo, X., Castillo-Ramírez, S., Reyes, A., Loza, M. G., et al. (2009). The Rhizobium etli RpoH1 and RpoH2 sigma factors are involved in different stress responses. Microbiology 155 , 386-397. doi: 10.1099/mic.0.021428-0

Mathews, S. A., Douglas, A., Sriprakash, K. S., and Hatch, T. P. (1993). In vitro transcription in Chlamydia psittaci and Chlamydia trachomatis. Mol. Microbiol. 7, 937-946. doi: 10.1111/j.1365-2958.1993.tb01185.x

Matsui, M., Takaya, A., and Yamamoto, T. (2008). 632 mediated negative regulation of Salmonella pathogenicity island 1 expression. J. Bacteriol. 190, 6636-6645. doi: 10.1128/JB.00744-08

mcClure, E. E., Chávez, A. S. O., Shaw, D. K., Carlyon, J. A., Ganta, R. R., Noh, S. M., et al. (2017). Engineering of obligate intracellular bacteria: progress, challenges and paradigms. Nat. Rev. Microbiol. 15, 544-558. doi: 10.1038/nrmicro. 2017.59

Meibom, K. L., Dubail, I., Dupuis, M., Barel, M., Lenco, J., Stulik, J., et al. (2008). The heat-shock protein ClpB of Francisella tularensis is involved in stress tolerance and is required for multiplication in target organs of infected mice. Mol. Microbiol. 67, 1384-1401. doi: 10.1111/j.1365-2958.2008. 06139.x

Mogk, A., Tomoyasu, T., Goloubinoff, P., Rüdiger, S., Röder, D., Langen, H., et al. (1999). Identification of thermolabile Escherichia coli proteins: prevention and reversion of aggregation by DnaK and ClpB. EMBO J. 18, 6934-6949. doi: 10.1093/emboj/18.24.6934

Mulligan, M. E., Brosius, J., and Mcclure, W. R. (1985). Characterization in vitro of the effect of spacer length on the activity of Escherichia coli RNA polymerase at the TAC promoter. J. Biol. Chem. 260, 3529-3538.

Nishihara, K., Kanemori, M., Kitagawa, M., Yanagi, H., and Yura, T. (1998). Chaperone coexpression plasmids: differential and synergistic roles of DnaKDnaJ-GrpE and GroEL-GroES in assisting folding of an allergen of Japanese cedar pollen, Cryj2, in Escherichia coli. Appl. Environ. Microbiol. 64, 1694-1699.

Nonaka, G., Blankschien, M., Herman, C., Gross, C. A., and Rhodius, V. A. (2006). Regulon and promoter analysis of the E. coli heat-shock factor, sigma32, reveals a multifaceted cellular response to heat stress. Genes Dev. 20, 1776-1789. doi: $10.1101 /$ gad. 1428206

Paget, M. S., and Helmann, J. D. (2003). The sigma70 family of sigma factors. Genome Biol. 4:203.

Rao, X., Deighan, P., Hua, Z., Hu, X., Wang, J., Luo, M., et al. (2009). A regulator from Chlamydia trachomatis modulates the activity of RNA polymerase through direct interaction with the beta subunit and the primary sigma subunit. Genes Dev. 23, 1818-1829. doi: 10.1101/gad.1784009

Rikihisa, Y. (2010). Anaplasma phagocytophilum and Ehrlichia chaffeensis: subversive manipulators of host cells. Nat. Rev. Micro. 8, 328-339. doi: 10.1038/ nrmicro2318

Rud, I., Jensen, P. R., Naterstad, K., and Axelsson, L. (2006). A synthetic promoter library for constitutive gene expression in Lactobacillus plantarum. Microbiology 152, 1011-1019. doi: $10.1099 / \mathrm{mic} .0 .28$ 599-0

Sahu, G. K., Chowdhury, R., and Das, J. (1994). Heat shock response and heat shock protein antigens of Vibrio cholerae. Infect. Immun. 62, 5624-5631.

Seo, G. M., Cheng, C., Tomich, J., and Ganta, R. R. (2008). Total, membrane, and immunogenic proteomes of macrophage- and tick cell-derived Ehrlichia chaffeensis evaluated by liquid chromatography-tandem mass spectrometry and
MALDI-TOF methods. Infect. Immun. 76, 4823-4832. doi: 10.1128/IAI.004 84-08

Shen, L., Li, M., and Zhang, Y. (2004). Chlamydia trachomatis $\sigma 28$ recognizes the fliC promoter of Escherichia coli and responds to heat shock in chlamydiae. Microbiology 150, 205-215. doi: 10.1099/mic.0.26734-0

Shimada, T., Yamazaki, Y., Tanaka, K., and Ishihama, A. (2014). The whole set of constitutive promoters recognized by RNA polymerase RpoD holoenzyme of Escherichia coli. PLoS One 9:e90447. doi: 10.1371/journal.pone.009 0447

Singh, S. S., Typas, A., Hengge, R., and Grainger, D. C. (2011). Escherichia coli sigma70 senses sequence and conformation of the promoter spacer region. Nucleic Acids Res. 39, 5109-5118. doi: 10.1093/nar/gkr080

Slamti, L., Livny, J., and Waldor, M. K. (2007). Global gene expression and phenotypic analysis of a Vibrio cholerae rpoH deletion mutant. J. Bacteriol. 189, 351-362. doi: 10.1128/jb.01297-06

Spector, M. P., and Kenyon, W. J. (2012). Resistance and survival strategies of Salmonella enterica to environmental stresses. Food Res. Int. 45, 455-481. doi: 10.1016/j.foodres.2011.06.056

Stoll, S., Feldhaar, H., and Gross, R. (2009). Promoter characterization in the at-rich genome of the obligate endosymbiont "Candidatus Blochmannia floridanus". J. Bacteriol. 191, 3747-3751. doi: 10.1128/JB.00069-09

Su, J., Yang, J., Zhao, D., Kawula, T. H., Banas, J. A., and Zhang, J.-R. (2007). Genome-wide identification of Francisella tularensis virulence determinants. Infect. Immun. 75, 3089-3101. doi: 10.1128/iai.01865-06

Tan, M., and Engel, J. N. (1996). Identification of sequences necessary for transcription in vitro from the Chlamydia trachomatis rRNA P1 promoter. J. Bacteriol. 178, 6975-6982. doi: 10.1128/jb.178.23.6975-6982. 1996

Tan, M., Gaal, T., Gourse, R. L., and Engel, J. N. (1998). Mutational analysis of the Chlamydia trachomatis rRNA P1 promoter defines four regions important for transcription in vitro. J. Bacteriol. 180, 2359-2366.

Tempel, R., Lai, X.-H., Crosa, L., Kozlowicz, B., and Heffron, F. (2006). Attenuated Francisella novicida transposon mutants protect mice against wildtype challenge. Infect. Immun. 74, 5095-5105. doi: 10.1128/iai.00598-06

Tripathi, L., Zhang, Y., and Lin, Z. (2014). Bacterial sigma factors as targets for engineered or synthetic transcriptional control. Front. Bioeng. Biotechnol. 2:33. doi: 10.3389/fbioe.2014.00033

Unver, A., Rikihisa, Y., Stich, R. W., Ohashi, N., and Felek, S. (2002). The omp-1 major outer membrane multigene family of Ehrlichia chaffeensis is differentially expressed in canine and tick hosts. Infect. Immun. 70, 4701-4704. doi: 10.1128/ iai.70.8.4701-4704.2002

Walker, D. H., and Dumler, J. S. (1996). Emergence of the ehrlichioses as human health problems. Emerg. Infect. Dis. 2, 18-29. doi: 10.3201/eid0201.960102

Walker, D. H., Paddock, C. D., and Dumler, J. S. (2008). Emerging and re-emerging tick-transmitted rickettsial and ehrlichial infections. Med. Clin. North Am. 92, 1345-1361. doi: 10.1016/j.mcna.2008.06.002

Wang, Y., and deHaseth, P. L. (2003). Sigma 32-dependent promoter activity in vivo: sequence determinants of the groE promoter. J. Bacteriol. 185, 5800-5806. doi: 10.1128/jb.185.19.5800-5806.2003

Wang, Y., Wei, L., Liu, H., Cheng, C., and Ganta, R. R. (2017). A genetic system for targeted mutations to disrupt and restore genes in the obligate bacterium, Ehrlichia chaffeensis. Sci. Rep. 7:15801. doi: 10.1038/s41598-01716023-y

Weiss, D. S., Brotcke, A., Henry, T., Margolis, J. J., Chan, K., and Monack, D. M. (2007). In vivo negative selection screen identifies genes required for Francisella virulence. Proc. Natl. Acad. Sci. U.S.A. 104, 6037-6042. doi: 10.1073/pnas. 0609675104

Wood, D. O., Wood, R. R., and Tucker, A. M. (2014). Genetic systems for studying obligate intracellular pathogens: an update. Curr. Opin. Microbiol. 17, 11-16. doi: 10.1016/j.mib.2013.10.006

Yabsley, M. J. (2010). Natural history of Ehrlichia chaffeensis: vertebrate hosts and tick vectors from the United States and evidence for endemic transmission in other countries. Vet. Parasitol. 167, 136-148. doi: 10.1016/j.vetpar.2009.09.015

Yu, H. H., and Tan, M. (2003). б28 RNA polymerase regulates hctB, a late developmental gene in Chlamydia. Mol. Microbiol. 50, 577-584. doi: 10.1046/j.1365-2958.2003.03708.x

Zhang, J. Z., Popov, V. L., Gao, S., Walker, D. H., and Yu, X. J. (2007). The developmental cycle of Ehrlichia chaffeensis in vertebrate 
cells. Cell Microbiol. 9, 610-618. doi: 10.1111/j.1462-5822.2006.00 812.x

Zhang, T., Kedzierska-Mieszkowska, S., Liu, H., Cheng, C., Ganta, R. R., and Zolkiewski, M. (2013). Aggregate-reactivation activity of the molecular chaperone ClpB from Ehrlichia chaffeensis. PLoS One 8:e62454. doi: 10.1371/journal.pone.0062454

Zhao, K., Liu, M., and Burgess, R. R. (2005). The global transcriptional response of Escherichia coli to induced sigma 32 protein involves sigma 32 regulon activation followed by inactivation and degradation of sigma 32 in vivo. J. Biol. Chem. 280, 17758-17768.
Conflict of Interest Statement: The authors declare that the research was conducted in the absence of any commercial or financial relationships that could be construed as a potential conflict of interest.

Copyright (c) 2019 Liu and Ganta. This is an open-access article distributed under the terms of the Creative Commons Attribution License (CC BY). The use, distribution or reproduction in other forums is permitted, provided the original author(s) and the copyright owner(s) are credited and that the original publication in this journal is cited, in accordance with accepted academic practice. No use, distribution or reproduction is permitted which does not comply with these terms. 